\title{
A Rare Case of Acute Abdomen
}

\author{
Dr.Rajeev Thilak C ${ }^{1}$, Dr.Ramadoss.P2, Dr.Ameesh Izath ${ }^{3}$, \\ Dr.Christianne Sandhya ${ }^{4}$. \\ 1,2,3,4 (Institue Of General Surgery, Madras Medical College, Chennai,India) ${ }^{1}$
}

\begin{abstract}
Ileum diverticulitis is a rare disease that may lead to an acute abdomen mimicking appendicitis. It is not Meckel diverticulitis nor other true diverticulum rather an acquired condition possibly due to the same causes as colonic diverticular disease ie pseudodiverticula. These diverticula are usually multiple and occur at the mesenteric border, sometimes hidden in the mesentery and overlooked during surgery. Most patients are asymptomatic and diagnosis is made on routine imaging studies or at autopsy. The acute complications of ileal diverticula including inflammation are very rare. The management of ileal diverticulitis is similar to that of colonic diverticulitis, i.e. treated conservatively if recognised on imaging prior to laparotomy. For those who present with complications such as bleeding, obstruction or perforation, surgical intervention is required. Our patient had a rare presentation of multiple ileal diverticula and in the antimesentric border which is very rare.
\end{abstract}

Keywords: Ileal diverticulum, acute abdomen, perforation

\section{Introduction}

Diverticulosis of ileum is uncommon. Diagnosis of small bowel diverticulitis is based on radiological findings rather than clinical findings. The main differential diagnosis of this condition includes acute appendicitis, or any other inflammatory bowel diseases such as crohn's disease. Complications include obstruction, bleeding and perforation. So preoperative knowledge of this condition may influence surgical and medical management in a easy way. Perforation is uncommon in diverticulum.

\section{Case Report}

$65 \mathrm{yr}$ old man presented to emergency department with progressively worsening diffuse abdominal pain for 1 day. There is no history of vomiting, fever, diahorroea, abdominal distension or urinary symptoms. There was no other comorbid illness for this patient. On examination he was afebrile, with tachycardia and hypotension. Diffuse abdominal pain with guarding and rigidity was present. Bowel sounds was absent. Perrectal examination show fecal staining+ .Laboratory investigations were essentially within normal range except slight elevation in WBC count. Xray abdomen shows pneumoperitoneum suggestive of hollow viscus perforation. The patient was resuscitated with intra venous fluids and higher antibiotics were given and taken immediately to the operating room for emergency laparotomy. A midline laparotomy incision was made and abdomen opened. It was noted that there was multiple diverticulum was present about $100 \mathrm{~cm}$ from the ileocaecal junction in the antimesenteric border and also there was a perforation in one of the diverticulum. The patient went complete resection of that segment and primary ileo-ileal anastomosis was done. The pathology result revealed Ileal diverticulitis. Postoperative period was uneventful and patient was discharged on the $12^{\text {th }}$ postoperative day.

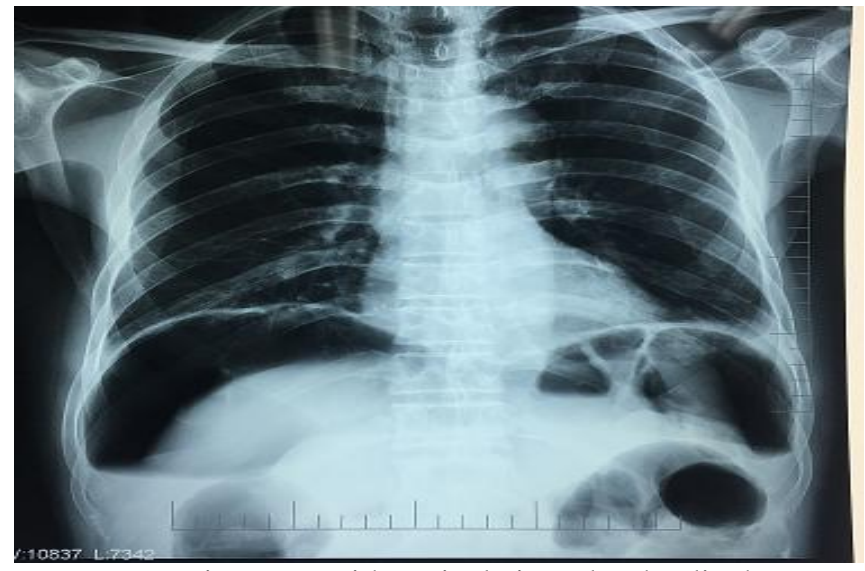

[Pneumoperitoneum with typical air under the diaphragm] 


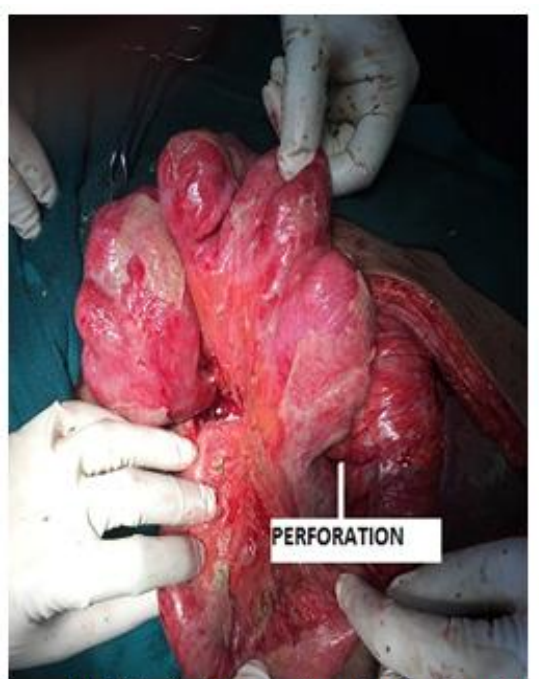

[Multiple diverticulum in the ileum]

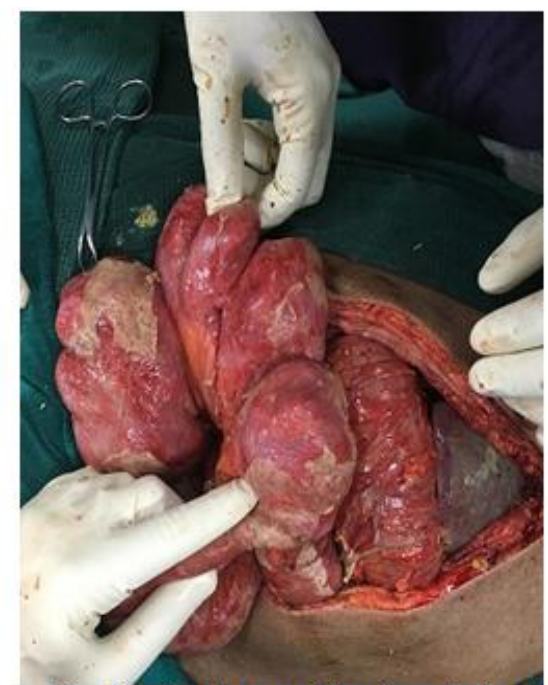

[perforation in one of the diverticulum]

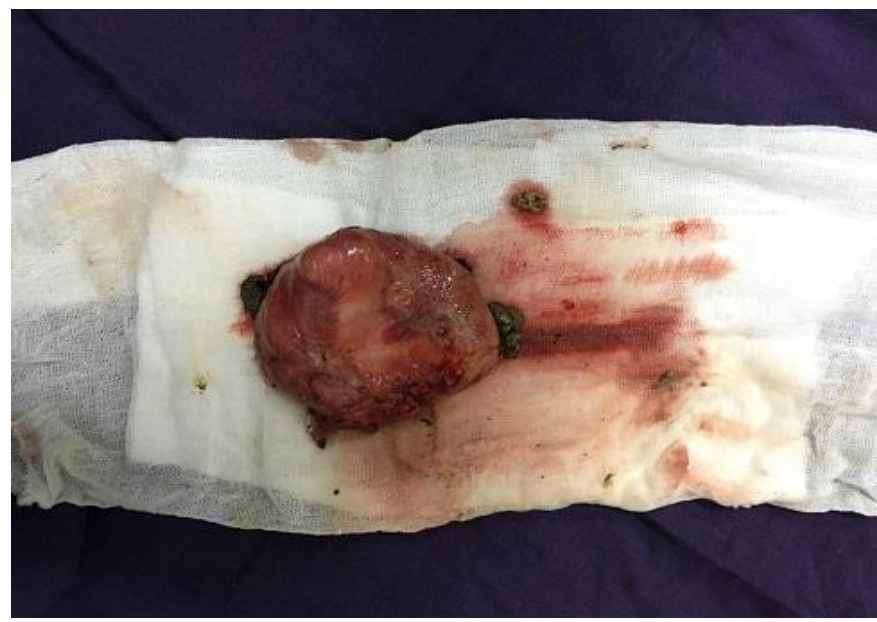

[Resected specimen]

\section{Discussion}

Incidence of ileal diverticulitis is about $0.1-1.5 \%{ }^{1}$. Ileal diverticula are false diverticula when compared with more common true meckel's diverticulum occurring in the ileum. Diverticulitis are usually multiple and present on the mesenteric border ${ }^{2}$.Diverticulosis is a condition of patients after sixth decade of life ${ }^{3}$. Acquired diverticula of ileum may be a primary condition or secondary due to abdominal surgery, tuberculosis or crohn's disease. Most patients are asymptomatic and diagnosis is made on routine imaging studies or only at autopsy. Patients who become symptomatic present with intermittent abdominal discomfort localizing to right lower quadrant. Complications include diverticulitis, intestinal obstruction, volvulus, bleeding/perforation. The acute complications are very rare occurring in only $6.5-10 \%$ of cases .The mortality of associated complications is $25-50 \%{ }^{4}$.

CT is the ideal investigation for ileal diverticula. Once a diagnosis of ileal diverticulitis is made, CT enterocyclisis has to be done to rule out any other small bowel diseases such as crohn's. Plain upright X-rays can also play a role. It may demonstrate a pneumoperitoneum suggestive of gastrointestinal perforation. Management of ileal diverticulitis is similar to that of colonic diverticulitis. It should be treated conservatively whenever possible with bowel rest, iv hydration, and iv antibiotics. For those who present with complications such as bleeding, perforation/obstruction surgical intervention is needed. The procedure includes segmental resection and primary anastomosis. Perforation in diverticulitis accounts for $2.3 \%-6.4 \%$ and it acoounts for 30 $50 \%$ of the complicated cases. Our case is rare as there were multiple ileal diverticula and were present on the anitmesentric border which presented with perforation. These findings are very uncommon.

\section{Conclusion}

Ileal diverticulitis although rare should be included as a differential in all cases of right lower quadrant pain .The index of suspicion should be high when CT findings show an inflammatory process but not suggestive 
of appendicitis .Simple cases are managed conservatively but complicated cases such as diverticular perforation require immediate surgical intervention in order to decrease morbidity and mortality.

\section{Acknowledgement}

I would like to thank Dr. Ramadoss.P M.S for his immense support and guidance. I would like to thank my institute, IGS, Madras Medical College for their support. Finally, my colleagues Dr.Ameesh Izath and Dr. Christianne Sandhya for their valuable assistance.

\section{References}

[1]. De Raet J, Brugman T, Geukens A. Non-Meckel's ileal diverticulitis with perforation: a rare cause of acute right lower quadrant pain. Acta Chir Belg. 2010;110:90-2.

[2]. Kirbas I, Yildirim E, Harman A, Basara O. Perforated ileal diverticulitis: CT findings. Diagn Interv Radiol. 2007;13:188-9.

[3]. Park H, Lee B. The management of terminal ileum diverticulitis. Am Surg. 2009;75:1199-202.

[4]. Wilcox RD, Shatney CH. Surgical significance of acquired ileal diverticulosis. Am Surg. 1990;56:222-5.

[5]. Makris K., Tsiotos G.G., Stafyla V., Sakorafas G.H. Small intestinal nonmeckelian diverticulosis. J. Clin. Gastroenterol. 2009;43:201-207

[6]. de Bree E., Grammatikakis J., Christodoulakis M., Tsiftsis D. The clinical significance of acquired jejunoileal diverticula. Am. J. Gastroenterol. 1998;93:2523-2528. 